\title{
Hints and Combined Constraints for First Generation Leptoquarks
}

\section{Luc Schnell ${ }^{a, b, c, d, *}$}

${ }^{a}$ Max Planck Institute for Physics,

Föhringer Ring 6, 80805 München, Germany

${ }^{b}$ Laboratoire de Physique Théorique et Hautes Énergies LPTHE, Sorbonne Université, CNRS, 4 place Jussieu, 75252 Paris Cedex 05, France

${ }^{c}$ Département de Physique, École Polytechnique, Route de Saclay, 91128 Palaiseau Cedex, France

${ }^{d}$ Departement Physik, ETH Zürich, Otto-Stern-Weg 1, 8093 Zürich, Switzerland

E-mail: schnell@mpp.mpg.de

We present a combined analysis of the existing hints for, and constraints on, the first generation fermion couplings of all ten leptoquark (LQ) representations. At the precision frontier we study the bounds from $K \rightarrow \pi \bar{v} v, K \rightarrow \pi \ell^{+} \ell^{-}, K^{0}-\bar{K}^{0}$ mixing, $D^{0}-\bar{D}^{0}$ mixing and parity violation experiments (APV and $Q_{\text {weak }}$ ), as well as a potential resolution of the Cabibbo Angle Anomaly (CAA) via LQ contributions to the $\beta$-decay. Although the CAA could be explained by the LQ representations $\Phi_{3}, V_{3}$, we observe that the necessary parameter space is excluded by other observables barring cancellations between different contributions. On the high-energy side we consider direct searches for single resonant production and pair production of LQs as well as Drell-Yan-like LQ signatures. Particular emphasis is placed on the recent CMS analysis of lepton flavor universality in non-resonant di-lepton pairs. The measured deviations from SM predictions could be a signature of $t$-channel contributions from $\tilde{\Phi}_{1}, \Phi_{2}, \Phi_{3}, \tilde{V}_{1}, V_{2}\left(\kappa_{2}^{R L} \neq 0\right)$ or $V_{3}$.

The Ninth Annual Conference on Large Hadron Collider Physics - LHCP2021

7-12 June 2021

Online

${ }^{*}$ Speaker 


\section{Introduction}

Leptoquarks (LQs) are hypothetical beyond the Standard Model (BSM) particles that couple to a quark and a lepton at tree-level [1]. In recent years they attracted wide attention since they can explain the flavor anomalies, deviations from Standard Model (SM) predictions that hint at BSM lepton flavor universality (LFU) violation. These flavor anomalies include the discrepancies in $R\left(D^{(*)}\right)$ [2-7], $b \rightarrow$ sl $\ell$ transitions [8-14] and the muon anomalous magnetic moment $a_{\mu}[15,16]$ with significances $>3 \sigma[17-21], \sim 6 \sigma[22,23]$ and $4.2 \sigma$ [24], respectively. They provide motivation to look for LQ effects in both precision and high-energy observables. We focus on LQs coupling to first generation fermions, since experimentally the sensitivity to these couplings is the highest and further anomalies emerged that can be traced back to first generation new physics (NP).

\subsection{Cabibbo-Angle-Anomaly (CAA)}

The first of these anomalies is the CAA, a deficit in first row CKM unitarity. Expressed analytically,

$$
\left|V_{u d}\right|^{2}+\left|V_{u s}\right|^{2}+\left|V_{u b}\right|^{2}=0.9985(5)
$$

was found to be smaller than unity [25], where $V$ is the CKM matrix. This could be explained via a first generation new physics (NP) contribution to super-allowed $\beta$-decays [26, 27], since the experimental value of $V_{u d}$ is determined based on this process. Using the SM hypothesis, one would then extract

$$
V_{u d}^{\beta}=V_{u d}\left(1+C_{11}^{e v_{e}}\right),
$$

instead of the unitary CKM matrix $V_{u d}$ of the Lagrangian alone, where $C_{11}^{e v e}$ quantifies the NP contribution as defined in Eq. (14) of Ref. [28]. If $C_{11}^{e v_{e}}$ has the right sign and magnitude, the CAA is resolved [27-31].

\subsection{Nonresonant Drell-Yan-like (DY-like) Production of Di-Leptons}

In addition, the CMS Collaboration recently published an analysis of nonresonant DY-like di-lepton events [32], finding values smaller than unity in the LFU ratio

$$
R_{\mu \mu / e e} \equiv \frac{d \sigma\left(p p \rightarrow \mu^{+} \mu^{-}\right) / d m_{\mu \mu}}{d \sigma\left(p p \rightarrow e^{+} e^{-}\right) / d m_{e e}},
$$

as shown by the black markers on the left of Fig. 2. The $\sim 4 \sigma$ deviation from SM predictions in the high invariant di-electron mass bin $\left(m_{\ell \ell}>1800 \mathrm{GeV}\right)$ also points towards LFU violating NP interacting with first generation fermions [27, 28].

\section{Setup}

We consider all ten possible LQ representations given in Table 1 and their possible couplings to the first generation weak eigenstates of the SM fermions. These are given by

$$
\begin{aligned}
\mathcal{L}_{\mathrm{LQ}}= & \lambda_{1}^{L} \bar{Q}^{c} i \tau_{2} L \Phi_{1}^{\dagger}+\lambda_{1}^{R} \bar{u}^{c} e \Phi_{1}^{\dagger}+\tilde{\lambda}_{1} \bar{d}^{c} e \tilde{\Phi}_{1}^{\dagger}+\lambda_{2}^{L R} \bar{Q} e \Phi_{2}+\lambda_{2}^{R L} \Phi_{2}^{T} \bar{u} i \tau_{2} L+\tilde{\lambda}_{2} \tilde{\Phi}_{2}^{T} \bar{d} i \tau_{2} L \\
& +\lambda_{3} \bar{Q}^{c} i \tau_{2}\left(\tau \cdot \Phi_{3}\right)^{\dagger} L+\kappa_{1}^{L} \bar{Q} \gamma_{\mu} L V_{1}^{\mu}+\kappa_{1}^{R} \bar{d} \gamma_{\mu} e V_{1}^{\mu}+\tilde{\kappa}_{1} \bar{u} \gamma_{\mu} e \tilde{V}_{1}^{\mu}+\kappa_{2}^{L R} \bar{Q}^{c} \gamma_{\mu} e V_{2}^{\mu \dagger} \\
& +\kappa_{2}^{R L} \bar{d}^{c} \gamma_{\mu} L V_{2}^{\mu \dagger}+\tilde{\kappa}_{2} \bar{u}^{c} \gamma_{\mu} L \tilde{V}_{2}^{\mu \dagger}+\kappa_{3} \bar{Q} \gamma_{\mu}\left(\tau \cdot V_{3}^{\mu}\right) L+\text { h.c. },
\end{aligned}
$$




\begin{tabular}{c|ccccc|ccccc} 
Field & $\Phi_{1}$ & $\tilde{\Phi}_{1}$ & $\Phi_{2}$ & $\tilde{\Phi}_{2}$ & $\Phi_{3}$ & $V_{1}$ & $\tilde{V}_{1}$ & $V_{2}$ & $\tilde{V}_{2}$ & $V_{3}$ \\
\hline$S U(3)_{c}$ & 3 & 3 & 3 & 3 & 3 & 3 & 3 & 3 & 3 & 3 \\
$S U(2)_{L}$ & 1 & 1 & 2 & 2 & 3 & 1 & 1 & 2 & 2 & 3 \\
$U(1)_{Y}$ & $-\frac{2}{3}$ & $-\frac{8}{3}$ & $\frac{7}{3}$ & $\frac{1}{3}$ & $-\frac{2}{3}$ & $\frac{4}{3}$ & $\frac{10}{3}$ & $-\frac{5}{3}$ & $\frac{1}{3}$ & $\frac{4}{3}$
\end{tabular}

Table 1: The ten possible representations of scalar and vector LQs under the SM gauge group.

where $Q$ and $L$ represent the left-handed first generation quark and lepton $S U(2)_{L}$ doublets, $e, d$ and $u$ the right-handed $S U(2)_{L}$ singlets, the superscript $c$ stands for charge conjugation and $\tau_{i}$ are the Pauli matrices. More information about our conventions for the LQ Lagrangian is given in Ref. [33]. After spontaneous EW symmetry breaking and the transition from the SM fermion weak eigenstates to the mass eigenstates, the first generation LQs also couple to the left-handed second and third generation fermion mass eigenstates [28]. These couplings are however Cabibbo-suppressed.

\section{Observables}

\subsection{Low-Energy Precision Observables}

At the low-energy precision frontier we analyze the LQ effects in $\beta$-decays that would explain the CAA, in parity violation experiments (PV) including APV [34, 35] and $Q_{\text {weak }}[36,37]$, in kaon decays including $K^{+} \rightarrow \pi^{+} \ell^{+} \ell^{-}$[38-40] and $K^{+} \rightarrow \pi^{+} \bar{v} v$ [41] as well as in meson mixing including $K^{0}-\bar{K}^{0}$ and $D^{0}-\bar{D}^{0}$ [42]. The corresponding diagrams are shown in the upper row of Fig. 1.

\subsection{High-Energy Direct Searches}

On the high-energy side, we consider the direct searches for pair production (PP) [43, 44], single production (SP) [45-47] and single resonant production (SRP) [48, 49] of LQs as well as the Drell-Yan-like (DY-like) production of di-leptons with LQ $t$-channel contributions [32, 47, 50, 51], displayed in the lower row of Fig. 1.

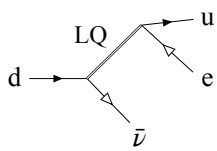

(CAA)

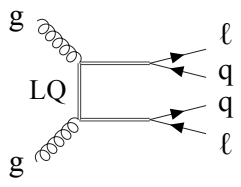

(PP)

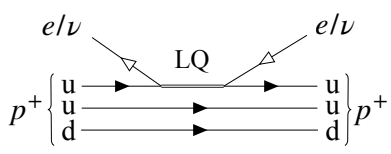

(PV)

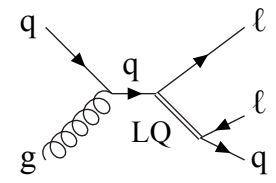

(SP)

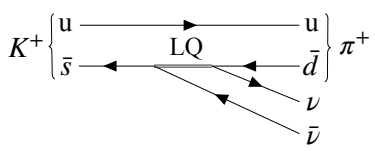

(kaon decays)

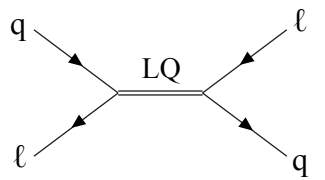

(SRP)

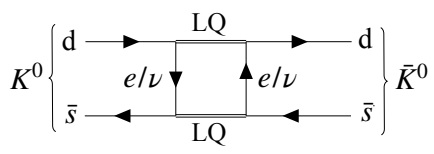

(meson mixing)

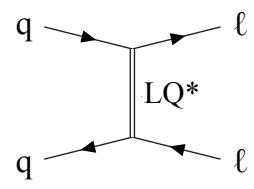

(DY-like)

Figure 1: Upper row: Feynman diagrams depicting the LQ contributions to the low-energy precision observables. The white arrows indicate charge-conjugate fermions, as explained in Appendix A of Ref. [33]. Lower row: Feynman diagrams for the LQ channels accessible to high-energy searches at LHC [28]. 

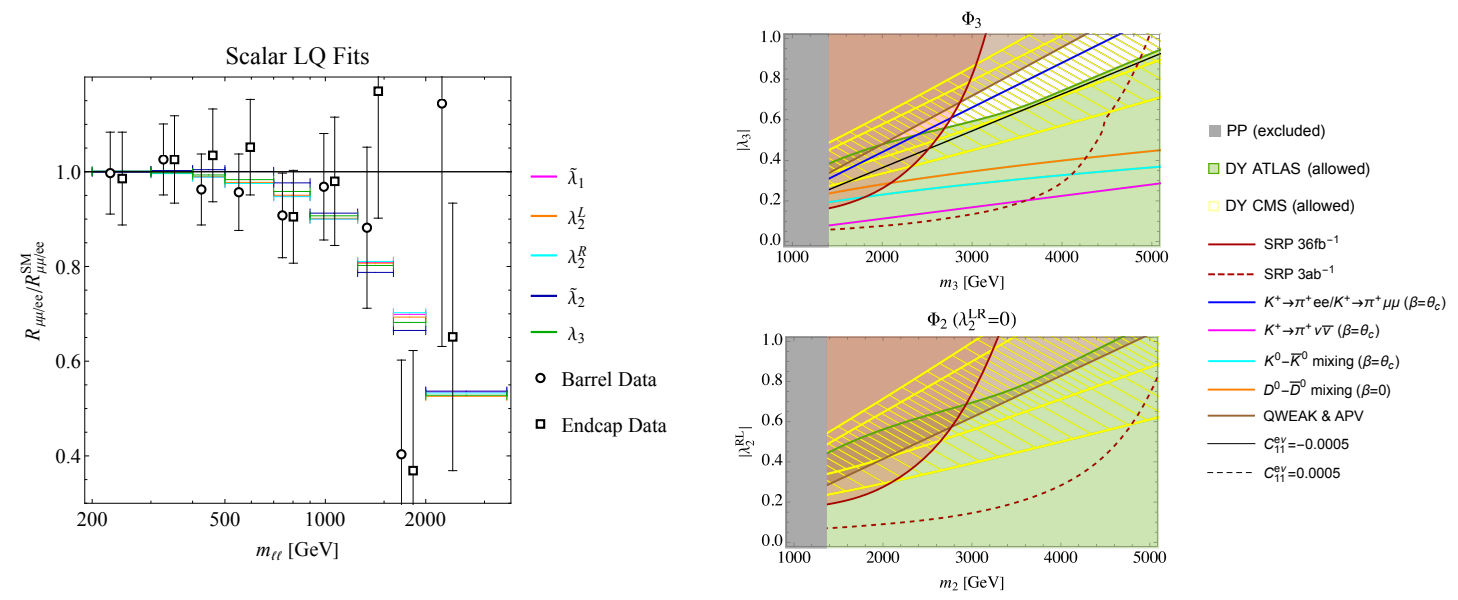

Figure 2: Left: The ratio $R_{\mu \mu / e e}^{\mathrm{Data}} / R_{\mu \mu / e e}^{\mathrm{MC}}$ measured by CMS [32], compared to $R_{\mu \mu / e e}^{\mathrm{SM}+\mathrm{LQ}} / R_{\mu \mu / e e}^{\mathrm{SM}}$ for the best fits of the various LQ models. The data points marked by circles (squares) correspond to measurements where none (at least one) of the leptons were detected in the CMS end caps. Right: Exclusion plots for the LQ representations $\Phi_{3}$ (top) and $\Phi_{2}\left(\lambda_{2}^{R L} \neq 0\right)$ (bottom) showing the contraints and preferred parameter regions from the low-energy precision observables and high-energy searches [28]. The parameter regions above the colored lines are excluded. We use the angle $\beta$ to distinguish between the up $\left(\beta=\theta_{c}\right)$ and down basis $(\beta=0)$ for the quarks, $\theta_{c}$ being the Cabibbo angle. See Ref. [28] for more information.

\section{Phenomenological Analysis}

On the left of Fig. 2, we show the best fits for the scalar first generation LQs to the CMS di-lepton data, indicated by the colored lines. The LQ models are preferred by $\sim 2.9 \sigma$ over the SM contribution alone [28]. On the right we show the limits and preferred regions in parameter space for $\Phi_{3}, \Phi_{2}\left(\lambda_{2}^{R L} \neq 0\right)$. Plots for the remaining LQ representations can be found in Ref. [28]. Both $\Phi_{3}, V_{3}$ can yield the right contribution to $C_{11}^{e v_{e}}$ in Eq. 2 to alleviate the CAA, the required parameter space for $\Phi_{3}$ is indicated by the black solid line. It is excluded both by the kaon decay (magenta) and the meson mixing (orange and cyan) limits. Although these depend on the angle $\beta$, they cannot be avoided simultaneously and, barring cancellations between different LQ couplings, they are therefore stringent. The same is true for $V_{3}$. The PV limits (brown) feature different levels of restrictiveness depending on the LQ representation, a detailed analysis of the PV limits is given in Ref. [52]. $\Phi_{2}\left(\lambda_{2}^{R L} \neq 0\right)$ features allowed parameter space that would explain the deviations from SM predictions measured by CMS (yellow). This is also true for the LQ representations $\tilde{\Phi}_{1}, \Phi_{2}\left(\lambda_{2}^{L R} \neq 0\right), \tilde{V}_{1}, V_{2}\left(\kappa_{2}^{R L} \neq 0\right)$ and $V_{3}$ that interfere constructively with the SM contribution.

\section{Conclusions}

We analyzed the sensitivity of different observables to first generation LQ couplings as well as the extent to which such models can explain the anomalies pointing towards LFU violation in the first generation. More data both at the high-energy and precision frontier is needed to consolidate the deviation from SM predictions in the DY-like di-lepton production and to potentially single out the LQ representation at its origin. We showed that a minimal explanation of the CAA via a single first generation LQ coupling is not viable. 


\section{References}

[1] W. Buchmuller, R. Ruckl, and D. Wyler, Phys. Lett. B 191, 442 (1987), [Erratum: Phys.Lett.B 448, 320-320 (1999)].

[2] J. P. Lees et al. (BaBar), Phys. Rev. Lett. 109, 101802 (2012), arXiv:1205.5442 [hep-ex] .

[3] J. P. Lees et al. (BaBar), Phys. Rev. D 88, 072012 (2013), arXiv:1303.0571 [hep-ex] .

[4] R. Aaij et al. (LHCb), Phys. Rev. Lett. 115, 111803 (2015), [Erratum: Phys.Rev.Lett. 115, 159901 (2015)], arXiv:1506.08614 [hep-ex] .

[5] R. Aaij et al. (LHCb), Phys. Rev. D 97, 072013 (2018), arXiv:1711.02505 [hep-ex] .

[6] R. Aaij et al. (LHCb), Phys. Rev. Lett. 120, 171802 (2018), arXiv:1708.08856 [hep-ex] .

[7] A. Abdesselam et al. (Belle), (2019), arXiv:1904.08794 [hep-ex] .

[8] V. Khachatryan et al. (CMS, LHCb), Nature 522, 68 (2015), arXiv:1411.4413 [hep-ex] .

[9] R. Aaij et al. (LHCb), JHEP 02, 104 (2016), arXiv:1512.04442 [hep-ex] .

[10] A. Abdesselam et al. (Belle), in LHC Ski 2016: A First Discussion of 13 TeV Results (2016) arXiv:1604.04042 [hep-ex] .

[11] R. Aaij et al. (LHCb), JHEP 08, 055 (2017), arXiv:1705.05802 [hep-ex] .

[12] R. Aaij et al. (LHCb), Phys. Rev. Lett. 122, 191801 (2019), arXiv:1903.09252 [hep-ex] .

[13] R. Aaij et al. (LHCb), Phys. Rev. Lett. 125, 011802 (2020), arXiv:2003.04831 [hep-ex] .

[14] R. Aaij et al. (LHCb), (2021), arXiv:2103.11769 [hep-ex] .

[15] G. W. Bennett et al. (Muon g-2), Phys. Rev. D 73, 072003 (2006), arXiv:hep-ex/0602035 .

[16] B. Abi et al. (Muon g-2), Phys. Rev. Lett. 126, 141801 (2021), arXiv:2104.03281 [hep-ex] .

[17] Y. Amhis et al. (HFLAV), Eur. Phys. J. C 77, 895 (2017), arXiv:1612.07233 [hep-ex] .

[18] C. Murgui, A. Peñuelas, M. Jung, and A. Pich, JHEP 09, 103 (2019), arXiv:1904.09311 [hep-ph] .

[19] R.-X. Shi, L.-S. Geng, B. Grinstein, S. Jäger, and J. Martin Camalich, JHEP 12, 065 (2019), arXiv:1905.08498 [hep-ph] .

[20] M. Blanke, A. Crivellin, T. Kitahara, M. Moscati, U. Nierste, and I. Nišandžić, (2019), 10.1103/PhysRevD.100.035035, [Addendum: Phys.Rev.D 100, 035035 (2019)], arXiv: 1905.08253 [hep-ph] .

[21] S. Kumbhakar, A. K. Alok, D. Kumar, and S. U. Sankar, PoS EPS-HEP2019, 272 (2020), arXiv:1909.02840 [hep-ph] . 
[22] W. Altmannshofer and P. Stangl, (2021), arXiv:2103.13370 [hep-ph] .

[23] M. Algueró, B. Capdevila, S. Descotes-Genon, J. Matias, and M. Novoa-Brunet, in 55th Rencontres de Moriond on QCD and High Energy Interactions (2021) arXiv:2104.08921 [hep-ph] .

[24] T. Aoyama et al., Phys. Rept. 887, 1 (2020), arXiv:2006.04822 [hep-ph] .

[25] P. A. Zyla et al. (Particle Data Group), PTEP 2020, 083 C01 (2020).

[26] A. Crivellin, M. Hoferichter, and C. A. Manzari, Phys. Rev. Lett. 127, 071801 (2021), arXiv:2102.02825 [hep-ph] .

[27] A. Crivellin, C. A. Manzari, and M. Montull, (2021), arXiv:2103.12003 [hep-ph] .

[28] A. Crivellin, D. Müller, and L. Schnell, Phys. Rev. D 103, 115023 (2021), arXiv:2104.06417 [hep-ph] .

[29] B. Belfatto, R. Beradze, and Z. Berezhiani, Eur. Phys. J. C 80, 149 (2020), arXiv:1906.02714 [hep-ph] .

[30] Y. Grossman, E. Passemar, and S. Schacht, JHEP 07, 068 (2020), arXiv:1911.07821 [hep-ph]

[31] C.-Y. Seng, X. Feng, M. Gorchtein, and L.-C. Jin, Phys. Rev. D 101, 111301 (2020), arXiv:2003.11264 [hep-ph] .

[32] A. M. Sirunyan et al. (CMS), JHEP 07, 208 (2021), arXiv:2103.02708 [hep-ex] .

[33] A. Crivellin and L. Schnell, (2021), arXiv:2105.04844 [hep-ph] .

[34] C. S. Wood, S. C. Bennett, D. Cho, B. P. Masterson, J. L. Roberts, C. E. Tanner, and C. E. Wieman, Science 275, 1759 (1997).

[35] V. A. Dzuba, J. C. Berengut, V. V. Flambaum, and B. Roberts, Phys. Rev. Lett. 109, 203003 (2012), arXiv:1207.5864 [hep-ph] .

[36] T. Allison et al. (Qweak), Nucl. Instrum. Meth. A 781, 105 (2015), arXiv:1409.7100 [physics.ins-det] .

[37] D. Androić et al. (Qweak), Nature 557, 207 (2018), arXiv:1905.08283 [nucl-ex] .

[38] J. R. Batley et al. (NA48/2), Phys. Lett. B 677, 246 (2009), arXiv:0903.3130 [hep-ex] .

[39] R. Appel et al. (E865), Phys. Rev. Lett. 83, 4482 (1999), arXiv:hep-ex/9907045 .

[40] J. R. Batley et al. (NA48/1), Phys. Lett. B 576, 43 (2003), arXiv:hep-ex/0309075 .

[41] A. V. Artamonov et al. (E949), Phys. Rev. Lett. 101, 191802 (2008), arXiv:0808.2459 [hep-ex] 
[42] M. Bona and L. Silvestrini (Utfit), PoS EPS-HEP2017, 205 (2017).

[43] A. M. Sirunyan et al. (CMS), Phys. Rev. D 98, 032005 (2018), arXiv:1805.10228 [hep-ex] .

[44] A. M. Sirunyan et al. (CMS), Phys. Rev. D 99, 052002 (2019), arXiv:1811.01197 [hep-ex] .

[45] V. Khachatryan et al. (CMS), Phys. Rev. D 93, 032005 (2016), [Erratum: Phys.Rev.D 95, 039906 (2017)], arXiv:1509.03750 [hep-ex] .

[46] T. Mandal, S. Mitra, and S. Seth, JHEP 07, 028 (2015), arXiv:1503.04689 [hep-ph] .

[47] M. Schmaltz and Y.-M. Zhong, JHEP 01, 132 (2019), arXiv:1810.10017 [hep-ph] .

[48] L. Buonocore, U. Haisch, P. Nason, F. Tramontano, and G. Zanderighi, Phys. Rev. Lett. 125, 231804 (2020), arXiv:2005.06475 [hep-ph] .

[49] A. Greljo and N. Selimovic, JHEP 03, 279 (2021), arXiv:2012.02092 [hep-ph] .

[50] A. M. Sirunyan et al. (CMS), JHEP 06, 120 (2018), arXiv:1803.06292 [hep-ex] .

[51] G. Aad et al. (ATLAS), JHEP 11, 005 (2020), [Erratum: JHEP 04, 142 (2021)], arXiv:2006.12946 [hep-ex] .

[52] A. Crivellin, M. Hoferichter, M. Kirk, C. A. Manzari, and L. Schnell, (2021), arXiv:2107.13569 [hep-ph] . 